\title{
Estudos sobre a memória na depressão: achados e implicações para a terapia cognitiva
}

\section{Studies on Memory in Depression: Findings and Implications for Cognitive Therapy}

\author{
Giovanni Kuckartz Pergher ${ }^{1}$ \\ LiLIAN MiLnitSKY STEIN ${ }^{2}$ \\ RICARDO WAINER ${ }^{3}$
}

\begin{abstract}
Resumo
O presente trabalho tem como objetivo apontar os benefícios decorrentes de uma integração entre pesquisa básica e psicoterapia. Enfatizaram-se as memórias autobiográficas de pacientes depressivos, as quais se caracterizam por serem supergeneralizadas (i. e., demasiadamente genéricas e inespecíficas). Dentre as conseqüências de uma tendência de processamento mnemônico supergeneralizado, estão a dificuldade para imaginar o futuro, déficits na habilidade de resolução de problemas, favorecimento da perpetuação de processos ruminativos e atos suicidas. As implicações para terapia cognitiva da depressão giram em torno da necessidade de se enfatizarem as especificidades, sejam elas a respeito de situações passadas ou de circunstâncias vindouras desejadas.
\end{abstract}

Palavras-chave : Depressão, memória, terapia cognitiva.

\begin{abstract}
The present study intends to point out the benefits of the integration between basic research and psychotherapy. Autobiographic memories of depressed patients were emphasized, which are characterized by being overgeneral (i. e., extremely generic and unespecific). Among the consequences of the tendency for overgeneral mnemonic processing there are the difficulties to ima-gine the future, deficits in problem solving skills, facilitation of the perpetuation of ruminative processes and suicidal acts. Implications for cognitive therapy on depression turns around the need to emphasize the specificity, whether they are related to past situations or to future desired circumstances.
\end{abstract} Keywords : Depression, memory, cognitive therapy.

1 Psicólogo mestrando em psicologia social e da personalidade pela Pontifícia Universidade Católica do Rio Grande Do Sul - Programa de Pós-graduação em Psicologia

2 Psicólogo, mestre e doutor em psicologia

$3 \mathrm{PhD}$ em psicologia

O presente trabalho recebeu apoio do CNPq.

Endereço para correspondência: Giovanni Kuckartz Pergher, Rua Ramiro Barcelos, 1450/403. 90035-002, Porto Alegre - RS, Brasil. Telefone: (51) 92462492, e-mail: gio@portoweb.com.br 
O amplo impacto que a Terapia Cognitiva (TC) causou no campo da psicologia clínica não se deu por acaso. Na mesma proporção em que foram sendo apresentados estudos empíricos comprobatórios de sua eficácia no tratamento de uma gama cada vez maior de transtornos mentais, esta modalidade psicoterápica foi conquistando seu espaço entre os profissionais da área da saúde mental (Beck e Freeman, 1990/1993).

Os resultados efetivos na terapêutica dos mais diferentes transtornos psiquiátricos, contudo, não se constituiu o único aspecto facilitador da ascensão da TC no cenário mundial. Além de eficácia comprovada, a TC conta com uma base teórica bastante consistente, caracterizando a prática desta abordagem de psicoterapia como uma atividade calcada em sólidos fundamentos. Os axiomas da teoria que sustenta a TC são robustos a ponto de possibilitar, no que se refere à aplicação, a implementação de técnicas oriundas originalmente de outras modalidades psicoterápicas, sem que se engendrem contradições, tanto em termos teóricos como epistemológicos (Beck e Alford, 1996/2000).

As teorizações que originalmente deram sustentação à TC foram sendo gradativamente construídas por seu idealizador, Aaron T. Beck, por volta do final dos anos 1950 e início dos anos 1960. Nessa época, Beck tinha como objetivo investigar os mecanismos inconscientes propostos pela psicanálise para explicação da depressão a partir de estudos empíricos e observações clínicas sistemáticas. Os resultados de suas investigações não se mostraram compatíveis com as pressuposições psicanalíticas, levando-o a buscar outros constructos que explicassem mais satisfatoriamente os dados empíricos observados (Beck e Alford, 1996/2000).

Beck, contudo, não estava inicialmente interessado em contemplar, em suas teorizações, explicações baseadas em achados experimentais que dessem conta dos diferentes processos cognitivos envolvidos nos transtornos psiquiátricos. Suas preocupações, ao invés disso, residiam em oferecer um modelo abrangente do funcionamento cognitivo (inicialmente de depressivos) como um todo, o qual apresentasse coerência interna e compatibilidade com as observações clínicas, possibilitando que novas intervenções psicoterápicas pudessem ser desenvolvidas (Beck, 1996). Para tanto, valeu-se de um método fundamentalmente abdutivo na construção de sua teoria, em que foram lançadas hipóteses mais gerais acerca do funcionamento cognitivo humano, cuja validade seria verificada através do sucesso terapêutico obtido pela implementação das estratégias construídas com base nas heurísticas clínicas formuladas.
Dessa forma, historicamente, houve desenvolvimentos praticamente independentes da TC, por um lado, e da psicologia cognitiva, por outro - muito embora houvesse plena compatibilidade entre conhecimentos construídos em cada um desses dois âmbitos (Wainer et al., no prelo). Em diversos momentos, foi apontada uma carência de integração entre o campo da pesquisa básica (i. e., pesquisas que visam a construir conhecimentos sobre um objeto de estudo específico, sem quaisquer comprometimentos com áreas aplicadas) e a psicoterapia. (Eysenck e Keane, 1989/1994).

Atualmente, existe um movimento de integração entre psicologia e TC, em que se busca unificar, na prática psicoterápica, refinamentos em termos explicativos acerca do funcionamento cognitivo nos mais diversos transtornos psiquiátricos (Eich e Schooler, 2000; Wainer, 1997, 2002). Tais sofisticações teóricas - construídas usualmente a partir de pesquisas experimentais - apresentam implicações no que tange a estratégias de intervenção, revelando um importante ganho que se obtém quando há uma prática de psicoterapia com sólidos fundamentos empíricos (Bootzin e McKnight, 1998).

Muitos seriam os transtornos mentais e os processos cognitivos que poderiam ser abordados em uma discussão acerca das contribuições de pesquisas experimentais para a prática psicoterápica (para uma revisão mais ampla veja Williams et al., 1997). No presente trabalho, entretanto, optou-se por focar determinadas relações existentes entre funcionamento mnemônico e depressão, bem como algumas implicações para TC desse transtorno psiquiátrico. Em especial, daremos ênfase às memórias autobiográficas (i. e., memórias que a pessoa possui sobre sua própria vida) de pacientes depressivos, área de investigação que tem atraído a atenção dos pesquisadores em função de suas implicações teóricas e terapêuticas.

Uma das características mais marcantes da memória autobiográfica de indivíduos com depressão é a de ser supergeneralizada, ou seja, uma recordação do próprio passado demasiadamente genérica, inespecífica e difusa (Williams et al., 1997). Pacientes com esse transtorno de humor, portanto, apresentam dificuldades para se lembrar de eventos específicos de sua história de vida, temporal e espacialmente localizados. As conseqüências desse modo de processamento mnemônico são discutidas a seguir, após apresentarmos os métodos experimentais utilizados em sua investigação, bem como algumas questões relativas à especificidade da supergeneralização nos transtornos depressivos. 
Em termos experimentais, as memórias autobiográficas supergeneralizadas são usualmente testadas através do Teste da Memória Autobiográfica (TMA), utilizado pela primeira vez em pacientes suicidas por Williams e Broadbent (1986). Nesse teste, são oferecidas dez palavras-estímulo para o participante, sendo cinco delas positivas (feliz, seguro, interessado, sucedido e surpreso) e cinco negativas (triste, irritado, desajeitado, ferido e solitário). As palavras são apresentadas uma de cada vez, alternando entre positivas e negativas. Os sujeitos são instruídos a se lembrarem de eventos específicos de sua vida que tenham relação com cada uma das palavras apresentadas. É explicitado que uma recordação específica é que aquela que possui localização temporal e espacial.

As respostas oferecidas para cada palavra-estímulo são então codificadas de acordo com três categorias, sendo que duas delas dizem respeito às memórias generalizadas e a outra concerne a memórias específicas. O primeiro tipo de memória generalizada é a categóri$\mathrm{ca}$, que se refere a eventos repetidos, sem qualquer referência a um tempo específico. Como exemplos, poderíamos citar "jantares românticos" ou "caminhadas na beira da praia”. O segundo tipo de recuperação generalizada é a estendida, na qual a lembrança remete a um determinado período de tempo, com início e fim determinados, porém com uma duração superior a um dia. "Minhas férias no Nordeste no ano passado" e "quando eu vivia no interior" poderiam ser exemplos deste tipo de memória estendida. Na terceira categoria para a classificação das memórias autobiográficas específicas, encontram-se as lembranças que possuem uma localização temporal específica com duração máxima de um dia. Exemplos deste tipo de recuperação poderiam ser "minha festa de formatura" ou "o dia em que conheci minha namorada".

Embora a observação de que indivíduos deprimidos apresentam uma tendência a processar a memória de maneira supergeneralizada venha sendo sistematicamente relatada na literatura (Healy e Williams, 1999), existem estudos em que tal tendência não é verificada (Dalgleish et al., 2001). A não-observação da supergeneralização em estudos que investigam transtornos específicos do humor - como o transtorno depressivo com padrão sazonal - sugere que o processamento mnemônico supergeneralizado não é uma característica universal dos transtornos afetivos. A literatura disponível no momento, entretanto, ainda não apresenta evidências suficientes que permitam distinguir os transtornos do humor em que a supergeneralização se mostra como uma característica mais marcante.
Também restam dúvidas no que diz respeito à estabilidade da supergeneralização. Em outras palavras, não se sabe ao certo se o processamento mnemônico supergeneralizado é dependente do humor atual ou se é uma característica presente tanto durante os episódios depressivos quanto nos períodos de remissão. Estudos de follow-up e pesquisas que comparam pacientes deprimidos com pacientes em remissão de episódio depressivo não apresentam resultados consistentes. Os estudos de follow-up, por um lado, apontam que a recuperação de memórias inespecíficas ocorre tanto durante o episódio depressivo quanto na remissão deste (Brittlebank et al., 1993; Peeters et al., 2002), dado que apóia a hipótese de que a supergeneralização é uma característica estável nos transtornos depressivos. Por outro lado, existem evidências de que a supergeneralização ocorre apenas na presença do humor deprimido. Wessel et al. (2001), ao compararem grupos de pacientes em episódio depressivo maior e pacientes em remissão, verificaram a supergeneralização apenas no primeiro grupo, sugestivo de que esse é processo dependente do humor. Embora os dados da literatura indiquem com maior consistência que a tendência de processamento mnemônico supergeneralizado é estável nos transtornos depressivos, esta ainda é uma questão que carece de investigação.

Um dado interessante apontado na literatura diz respeito à particularidade do processo de supergeneralização nos transtornos depressivos. Estudos que utilizaram o TMA para investigar a qualidade da memória em outros transtornos, como transtorno de ansiedade generalizada (Burke e Mathews, 1992), transtorno obsessivo-compulsivo (Wilhelm et al., 1997) e transtorno de personalidade borderline (Arntz et al., 2002) não detectaram com sistematização uma propensão à recuperação de memórias inespecíficas, a não ser que houvesse sintomas depressivos comórbidos (Wessel et al., 2001). Uma exceção é verificada apenas para o transtorno de estresse pós-traumático e transtorno de estresse agudo, nos quais a supergeneralização é verificada mais consistentemente (Harvey et al., 1998; McNally et al., 1994; McNally et al., 1995). Possivelmente, um mecanismo de evitação de memórias dolorosas esteja envolvido tanto nos transtornos depressivos quanto nos transtornos desencadeados por traumas. Uma discussão mais aprofundada sobre esse tema, contudo, foge aos objetivos do presente artigo. 


\section{Memória e imaginação do futuro}

Quando se fala em memória, a maioria das pessoas faz associações com questões relacionadas ao passado - pensam no sistema mnemônico humano como um armazenador que grava experiências anteriores no intuito de utilizá-las no presente. Poucos se dão conta, contudo, do papel essencial que a memória tem na construção de expectativas com relação ao futuro (Neufeld e Stein, 2001). Em outras palavras, aquilo que esperamos por vir possui íntima relação com o que já experenciamos, uma vez que as memórias a respeito de nosso próprio passado constituem-se um fundamento para elaborarmos uma visão de nosso futuro (Tulving e Lepage, 2000).

Considere perguntas como: "a que horas você vai chegar em casa hoje?", ou então "semana que vem você tem um tempo para irmos ao cinema?". Para respondêlas, é preciso que cenários futuros sejam elaborados em nossas mentes e, para isso, a recuperação de eventos anteriores é imprescindível. A lembrança de situações passadas será responsável pela disponibilização de dados objetivos que servirão como base para construção dos cenários vindouros (Williams et al., 1996).

O fato de as lembranças de nosso passado influenciarem diretamente uma projeção daquilo que está por vir possui uma série de implicações. Uma delas e talvez uma das mais importantes - concerne aos objetivos que traçamos para nossas vidas. Ao estabelecermos planos e metas, sejam elas na esfera profissional, interpessoal, familiar etc., usualmente o fazemos no intuito de efetivamente alcançá-las e, para tanto, nos valemos de experiências anteriores, as quais fornecem as bases para pensarmos naquilo que podemos ou não esperar (Schacter, 2001).

Quando as memórias da pessoa a respeito de seu próprio passado são genéricas e inespecíficas em demasia - tal qual ocorre na depressão - a visualização de eventos vindouros pode ser comprometida. A hipótese de que as memórias autobiográficas supergeneralizadas dificultam a elaboração de cenários futuros foi testada por Williams et al. (1996). Em uma série de três experimentos, os autores compararam o desempenho de indivíduos com história de tentativa de suicídio (dos 24 participantes que compunham este grupo, 20 satisfaziam critérios para transtorno depressivo) com indivíduos-controle sem diagnóstico de depressão em dois tipos de testes. O primeiro deles era o TMA, havendo apenas algumas modificações experimentais dependendo do experimento. O segundo, utilizado para investigação da imaginação de cená- rios futuros, envolvia os mesmos princípios gerais do TMA, porém os participantes deveriam utilizar as palavras-estímulo para figurarem eventos vindouros que ainda não haviam acontecido com eles.

Conforme o esperado, os resultados indicaram que, quanto mais genéricas eram as memórias autobiográficas recuperadas pelos participantes, menos específicos eram os cenários futuros imaginados. Os resultados também apontaram que tais cenários poderiam tornar-se mais específicos se os participantes fossem estimulados a buscar lembranças episódicas mais detalhadas (Williams et al., 1996).

Na terapia cognitiva da depressão, grande parte do trabalho psicoterápico é norteado por metas que são conjuntamente estabelecidas entre paciente e terapeuta. Essas metas, para otimização da terapia, devem ser elaboradas em termos mais objetivos possíveis, de modo que possam ser determinados passos para sua consecução e que os progressos possam ser avaliados com critérios mais precisos (Beck et al., 1979/1997). Nesse sentido, os psicoterapeutas devem estar atentos para a qualidade da memória autobiográfica de seus pacientes, pois, caso esta se caracterize por ser supergeneralizada, há um obstáculo em potencial para o estabelecimento de metas para o tratamento.

Além disso, é consenso entre os terapeutas cognitivos que o suicídio está fortemente relacionado com uma visão negativa do futuro, também chamada de desesperança. Se pensarmos nessa falta de perspectivas quanto ao futuro como tendo parte de seus fundamentos em uma recuperação nebulosa do passado, seria atribuído aos terapeutas desconsiderar o papel exercido pelas memórias autobiográficas supergeneralizadas na abordagem do comportamento e ideação suicida de seus pacientes. Voltaremos mais adiante à questão do suicídio após analisarmos outras implicações do processamento mnemônico supergeneralizado na depressão.

\section{Memória e resolução de problemas}

Uma das mais importantes funções cognitivas afetadas pela depressão é a resolução de problemas (Nezu e Nezu, 2001; Wainer, 1997). Isso tem uma série de conseqüências negativas para o indivíduo acometido por esse transtorno de humor, principalmente se considerarmos a enorme gama de problemas com os quais nos defrontamos em nosso cotidiano. Questões tais como "qual ônibus devo pegar para chegar àquele 
shopping na zona norte da cidade?" ou "meu telefone está mudo e hoje tenho que ligar para clientes importantes - o que fazer?" revelam as constantes demandas do dia-a-dia por nossas habilidades de resolver problemas. Quando essas habilidades apresentam déficits - tal qual comumente ocorre com pessoas que sofrem de depressão - são grandes as chances de o indivíduo tornar-se desadaptado ao meio no qual está inserido.

Os déficits na capacidade de resolver problemas acabam por contribuir com a perpetuação da depressão, pois muitas vezes a pessoa apresenta fracassos reais no manejo de situações cotidianas, que acabam por reforçar suas idéias de incapacidade e menos valia (Young et al., 1993/1999). A resolução de problemas, portanto, tem se mostrado como importante foco a ser trabalhado na terapia cognitiva da depressão (Beck, 1995/1997).

No âmbito da psicologia cognitiva, esforços têm sido realizados na geração de modelos explicativos que auxiliem na compreensão dos mecanismos psicológicos envolvidos no processo de resolução de problemas. Um desses modelos, proposto por Sternberg (1996/ 2000), sugere que o ciclo de resolução de problemas envolve sete etapas, quais sejam: 1) identificação do problema; 2) definição do problema; 3) construção de uma estratégia para resolução do problema; 4) organização de informação sobre o problema; 5) alocação de recursos; 6) monitorização da resolução do problema e 7) avaliação da resolução do problema. Os processos mnemônicos desempenham seu mais importante papel nas primeiras etapas do ciclo, referentes à definição do problema e formulação de estratégias (Evans et al., 1992). Desta forma, se os mecanismos de codificação e recuperação de memória envolvidos nessas etapas iniciais se mostrarem enviesados, é razoável supor que todo o restante do ciclo de resolução de problemas acaba comprometido.

A hipótese de que uma tendência de processamento mnemônico supergeneralizada dificulta uma resolução efetiva de problemas em pacientes depressivos foi avaliada por Goddard et al. (1996). Em seu estudo, participantes com diagnóstico de transtorno depressivo maior e participantes-controle sem este diagnóstico realizaram o TMA e, posteriormente, foram submetidos a um procedimento denominado "Solução de Problemas Meios-Fim” (SPMF).

No SPMF, foi apresentada uma situação-problema que possuía um estado inicial e um estado final desejado. A tarefa do participante consistia em descrever os meios pelos quais ele seguiria para chegar ao estado final desejado. Um exemplo desse tipo de situação é o seguinte: "Você percebe que seus amigos parecem estar evitando-o. Você quer ter amigos e ser benquisto. A história termina quando seus amigos voltam a gostar de você. Portanto, a situação inicia quando você percebe pela primeira vez que seus amigos o estão evitando".

As respostas dos sujeitos concernentes às ações que tomariam para o alcance dos objetivos foram então classificadas de acordo com a sua efetividade. Para tanto, foi utilizada uma escala Likert de oito pontos, que variava de "nada efetiva" (0) até "muito efetiva" (7). Além disso, no experimento de Goddard et al. (1996), os participantes foram instruídos a relatar quaisquer memórias e/ou pensamentos que lhes viessem à cabeça durante o processo de construção de estratégias de solução. Desta forma, havia dois momentos em que os participantes tinham a tarefa de recuperar memórias: uma no início do experimento através do TMA e outra no decorrer da realização do SPMF.

Genericamente falando, os resultados de Goddard et al. (1996) indicaram que a habilidade para recuperar memórias específicas no TMA estava positivamente relacionada com a performance no procedimento de solução de problemas meios-fim, ou seja, quanto mais memórias específicas eram recuperadas no TMA, mais soluções possíveis eram relatadas no SPMF. Contudo, foi encontrada uma relação muito fraca entre a recuperação de memórias autobiográficas supergeneralizadas no TMA (no início do experimento) e a geração de respostas efetivas no procedimento de resolução de problemas. Por outro lado, especialmente para o grupo de depressivos, foi encontrada uma significativa associação entre a recuperação de memórias genéricas durante a geração de soluções e a inefetividade destas.

A ausência de associação significativa entre a recuperação categórica no TMA e o mau desempenho no SPMF sugere que a inabilidade em recuperar memórias específicas, em si, não é a única responsável pelos déficits de resolução de problemas. Se este fosse o caso, aqueles que geraram memórias autobiográficas supergeneralizadas por ocasião do TMA necessariamente deveriam apresentar pior performance no SPMF. A recuperação categórica, entretanto, parece exercer sua influência através de outros mecanismos, pois o processo de resolução de problemas fica comprometido somente quando o estilo de recuperação supergeneralizado faz-se presente no momento da definição do problema e busca de soluções.

Tomados em conjunto, os resultados de Goddard et al. (1996) indicam que os processos mnemônicos 
estão envolvidos direta e indiretamente com a capacidade para resolver problemas. Diretamente, foram evidenciadas relações significativas entre a habilidade em gerar recordações específicas no TMA e o desempenho no SPMF. Teoricamente, esse resultado sugere que a disponibilidade de um "banco de dados" com informações precisas e detalhadas constitui-se um importante componente para a produção de soluções efetivas aos problemas apresentados.

Um efeito indireto dos processos mnemônicos sobre a resolução também foi verificado, pois não foi detectada uma associação relevante entre um padrão de recuperação supergeneralizado em si (mensurado através do TMA) e a inefetividade das soluções apresentadas. Conforme sugerido pelas autoras, um estilo de recuperação categórico durante o processo de resolução de problemas pode favorecer o estabelecimento de um padrão ruminativo, que maximiza a preocupação com fracassos passados e reduz os recursos cognitivos necessários para o engajamento em um pensamento mais produtivo.

A ruminação pode ser entendida como pensamentos e comportamentos que tornam a atenção da pessoa focada em seus próprios sintomas depressivos, bem como nas conseqüências indesejáveis destes (NolenHoeksema, 1991). Em decorrência desse estilo de pensamento analítico (i. é., que faz julgamentos) e autocentrado, com foco incidindo fundamentalmente sobre emoções negativas, diminui a propensão da pessoa em engajar-se em pensamentos e comportamentos mais adaptativos que possam efetivamente auxiliar no enfrentamento dos incapacitantes sintomas depressivos (Lyubomirsky e Nolen-Hoeksema, 1995).

Em termos de implicações para a terapia cognitiva da depressão, reforça-se a necessidade de ser considerada a inespecificidade da memória autobiográfica de pacientes depressivos ao trabalhar-se com técnicas de resolução de problemas (Pollock e Williams, 2001). Estratégias objetivas para lidar com uma tendência de processamento mnemônico supergeneralizado serão abordadas na sessão final, ao discutirmos a utilização dos conhecimentos advindos de pesquisas experimentais sobre o funcionamento mnemônico depressivo no âmbito da psicoterapia.

\section{Memória e suicídio}

O risco de suicídio iminente é sem dúvida uma das situações mais delicadas com que o psicoterapeuta pode se deparar em sua prática clínica. Por razões óbvias, a ideação suicida, quando detectada, requer avaliação e intervenção imediata. Para que esta seja a mais precisa e eficaz possível, o terapeuta deve conhecer os complexos mecanismos envolvidos nas intenções em pôr fim à própria vida. Dentre os diversos fatores associados ao suicídio, as memórias autobiográficas supergeneralizadas têm sido consideradas como exercendo papel relevante (Williams e Wells, 1992,1994).

O estudo clássico de Williams e Broadbent (1986) sobre as memórias autobiográficas supergeneralizadas, que impulsionou grande parte dos estudos subseqüentes na área, investigou justamente o processo de recuperação categórica em pacientes suicidas. Em seus experimentos, participantes com história de tentativa de suicídio e participantes-controle foram submetidos ao TMA e a outros testes para mensuração de sintomas depressivos e desesperança. Conforme o esperado, aqueles pacientes que haviam tido tentativas de suicídio prévias apresentaram maiores níveis de desesperança e maiores índices de recuperação categórica de memórias autobiográficas. Um entendimento mais aprofundado acerca da relação entre uma tendência de processamento mnemônico supergeneralizado e suicídio foi se constituindo nos anos seguintes, de modo que procuraremos abordar alguns dos mecanismos sugeridos como mediadores dessa relação.

Já foi colocado anteriormente que é com base no passado que fazemos nossas interpretações e inferências acerca do futuro, bem como que a visão negativa de futuro (desesperança) está intimamente relacionada com a ideação e comportamento suicida. Se a recuperação de nosso passado for específica, poderemos imaginar um futuro também em termos específicos. Considere o caso de uma pessoa que está saindo do colégio para ingressar na faculdade. Ela pode ter muitas lembranças de maus desempenhos anteriores, tais como: "naquela prova trimestral de matemática no final do segundo ano tive uma péssima nota, mas lembro que o rompimento de um namoro na época me dificultou bastante o engajamento nos estudos. Depois disso, consegui fazer a prova de recuperação e passei de ano, com direito a festa de comemoração com meus colegas". Nesta descrição, evidencia-se bastante especificidade da recordação, o que possivelmente contribuiria para que os tempos vindouros de faculdade fossem concebidos em termos de potenciais dificuldades, as quais, contudo, não são insuperáveis e podem oferecer saborosas recompensas.

Suponha, por outro lado, que a pessoa que está para entrar no ensino superior recupere seu passado da seguinte forma: "sempre fui um fracasso e os estu- 
dos só me trouxeram desgosto". Neste caso, principalmente se o indivíduo estiver com um humor significativamente deprimido, suas inferências e interpretações sobre o próprio futuro possuem grandes chances de serem catastróficas, caracterizadas por uma inespecificidade e nebulosidade negativamente carregada. Na perspectiva do paciente, o suicídio pode ser a única forma de escape do futuro tão desesperador que o espera.

As memórias autobiográficas supergeneralizadas também contribuem através de outros mecanismos para o surgimento da ideação e atitudes suicidas. Basicamente, os déficits reais e percebidos quanto à capacidade de resolução de problemas, associados à ruminação, acabam por gerar uma perspectiva de impossibilidade de mudança (Pollock e Williams, 1998). Quando o indivíduo falha em acessar memórias específicas, ficam aumentadas as chances de se estabelecerem processos ruminativos (por exemplo, ficar repetindo para si mesmo a mesma idéia geral "estou mal, estou mal, estou mal...”) (Jones, 1999), e diminuídos os recursos disponíveis para lançar mão de soluções efetivas para as dificuldades (Pollock e Williams, 2001). A partir disto, o depressivo enxerga sua degradante condição como intransponível e imutável, a menos, é claro, que ele decrete o final do sofrimento através do fim da própria vida.

\section{Implicações para terapia cognitiva}

Até o presente momento, focalizamos nossa discussão nas implicações que uma tendência de processamento mnemônico supergeneralizado possui na gênese e manutenção dos estados depressivos. Em linhas gerais, apontamos que as memórias autobiográficas prejudicam a capacidade do indivíduo para imaginar seu futuro, provocam déficits nas habilidades de resolução de problemas e favorecem a perpetuação de processos ruminativos, sendo, portanto, um importante componente na ideação e comportamento suicida. A partir de agora, abordaremos estratégias de intervenção psicoterápica que visam a contornar as tendenciosidades negativas da memória de depressivos. Essas intervenções, vale ressaltar, tiveram seu desenvolvimento e aperfeiçoamento a partir dos conhecimentos advindos de pesquisas experimentais.

Embora a propensão para recuperar memórias autobiográficas de maneira supergeneralizada seja considerada como uma característica estável dos depressivos (Brittlebank et al., 1993), existem evidências consistentes de que ela é sujeita a modificações, pelo menos em curto prazo (Watkins et al., 2000; Watkins e Teasdale, 2001; Williams et al., 2000). Os indícios empíricos quanto à possibilidade de haver mudanças no padrão de processamento mnemônico supergeneralizado são de suma importância, uma vez que oferecem bases sólidas que justificam o engajamento dos psicoterapeutas em técnicas para trabalhar esse mecanismo cognitivo perpetuador da depressão com seus pacientes.

Uma das premissas básicas da terapia cognitiva é a de que os indivíduos não sofrem pelos eventos em si, mas sim pela interpretação que fazem desses eventos. Dessa forma, um objetivo básico da terapia cognitiva é o de auxiliar os pacientes a buscarem interpretações alternativas para as situações que vivenciam. Para que interpretações alternativas possam ser satisfatoriamente consideradas, é preciso que se tenha acesso a especificidades das situações em questão. Caso os eventos sejam recuperados de forma demasiadamente genérica, fica dificultada a busca por diferentes interpretações para eles (Williams, 1996).

No intuito de recrudescer as habilidades dos pacientes em codificar e recuperar os eventos de maneira mais específica, uma poderosa ferramenta a ser posta em prática pelos terapeutas envolve o emprego de diários. A partir do uso de diários, os pacientes são estimulados a atentarem para uma quantidade maior de detalhes das situações, favorecendo para que estas sejam posteriormente avaliadas de maneira mais racional. Ao lançar mão da utilização de diários, os terapeutas devem ser cuidadosos ao instruírem seus pacientes a serem os mais específicos que puderem, pois, caso não façam esta recomendação, corre-se o risco de o diário tornar-se um caderno de anotações vagas, baseadas em memórias inespecíficas, as quais possuem pouco valor terapêutico (Williams, 1992).

Os terapeutas também devem atentar para não considerar de imediato a não-conclusão dos registros diários como uma forma de resistência ou de boicote ao tratamento. Tendo em vista a quase incapacidade de indivíduos depressivos de acessar memórias autobiográficas específicas, não é de se estranhar que enxerguem a utilização do diário como uma tarefa com alto nível de dificuldade. Não conseguir fazer um registro ao final do dia pode inclusive ser interpretado pelo paciente como mais um indicativo de sua ampla incompetência. Nesse sentido, os psicoterapeutas podem contornar esse empecilho ao sugerir a realização das anotações em espaços de tempo menores, como turnos ou até mesmo ao final de cada hora. 
O encorajamento à codificação e recuperação de maior quantidade de detalhes pode ser feito através de estratégias de anamnese. $\mathrm{Na}$ "terapia anamnésica” (Williams, 1992), o terapeuta guia uma recuperação deliberada de eventos passados vivenciados por seus pacientes. A partir de pistas como atividades, pessoas, lugares etc., o paciente é estimulado a examinar sua memória com maior veemência, procurando por especificidades das circunstâncias. Essa busca por detalhes, vale ressaltar, deve ser realizada tanto para eventos positivos quanto negativos. No concernente aos positivos, uma recuperação detalhada pode auxiliar na melhoria do estado de humor, ao mesmo tempo em que propicia o acesso a um "banco de dados" mais rico para utilização na resolução de problemas. No que tange aos eventos negativos, a busca por especificidades ajuda a evitar que o paciente faça generalizações depreciativas, dificultando o processo comum de auto-imposição de rótulos abrangentes, tais como "sou um fracasso" ou "sou indesejável".

As estratégias de resolução de problemas - amplamente utilizadas na terapia cognitiva da depressão - devem levar em consideração a questão da supergeneralização (Pollock e Williams, 2001). No momento em que se define um problema a ser enfrentado, uma meta é estabelecida. Para que esta possa ser alcançada, em primeiro lugar, é crucial que a meta seja conceitualizada em termos mais precisos possíveis. Quando um objetivo é posto de maneira vaga (por exemplo "ser feliz", "sair dessa”) - que é a tendência natural dos depressivos em função de seu padrão de processamento mnemônico supergeneralizado - os passos para alcançá-lo não podem ser definidos com clareza, impedindo sua consecução. Nesse sentido, os psicoterapeutas devem estar atentos para, num primeiro momento do trabalho de resolução de problemas, dedicarem o tempo que for necessário para definirem o problema com a maior exatidão possível (Wainer et al., 2003).

Quando o trabalho de resolução de problemas já estiver na etapa de construção de uma estratégia, os pacientes igualmente devem ser instigados a serem específicos. Para tanto, o terapeuta pode estimular o paciente a buscar situações análogas em seu passado, procurando pela maior quantidade possível de informações que possam oferecer suporte para a formulação de estratégias de resolução. Novamente, cabe ao terapeuta guiar esse processo de procura, pois, se depender única e exclusivamente da memória do paciente depressivo, não serão recuperadas informações re- levantes suficientes que possam efetivamente auxiliar na resolução do problema em questão. Assim, indagações tais como "quais eram as circunstâncias em que aquela situação ocorreu?”, “quem estava envolvido?” e "que tempos eram aqueles, e em que eles divergiam dos de hoje?" podem ajudar para que importantes elementos sejam acessados e utilizados na situação problemática atual. Além disso, esse processo de busca incita o paciente a colocar-se em uma postura ativa no tratamento, pois que, com o treino, vai adquirindo habilidades de acessar um "banco de dados" relevante por conta própria, passando para uma resolução de seus problemas cada vez com maior autonomia (D’Zurilla, 1988).

A questão do suicídio também pode ser abordada a partir de intervenções que levam em conta a tendência de processamento mnemônico supergeneralizado. Uma das estratégias utilizadas com pacientes suicidas consiste na análise de razões para morrer versus razões para viver. Quando da utilização desta estratégia, os pacientes usualmente encontram maior facilidade no relato das razões pelas quais desejariam a própria morte. O outro lado, das razões para viver, é dificilmente bem desenvolvido pelos pacientes em função, entre outras coisas, de sua habilidade para imaginar o futuro estar prejudicada.

Os terapeutas podem maximizar a eficácia da estratégia de análise de razões de morrer versus razões para viver através de técnicas de imaginação do futuro. Nestas técnicas, os pacientes são convidados a visualizar, da forma mais detalhada possível, como seria seu futuro daqui a cinco minutos, cinco horas, cinco dias, cinco semanas, cinco meses, e assim por diante. Conforme ressaltado por Williams e Wells (1992/1994), o interesse maior não reside em uma busca de concretização do futuro imaginado, mas sim em dar uma perspectiva de continuidade ao paciente. Com isso, há uma redução da desesperança e, assim, uma redução da probabilidade de ocorrência de um ato suicida.

Por fim, ressalta-se a necessidade de os clínicos considerarem a questão do funcionamento mnemônico dos depressivos em suas intervenções, principalmente no que concerne à qualidade das memórias recuperadas dentro e fora do setting terapêutico. Em especial, devem atentar não só para o que é lembrado, dando maior ênfase para como é lembrado (Williams, 1996). 


\section{Referências bibliográficas}

Arntz, A.; Meeren, M.; Wessel, I. - No Evidence for Overgeneral Memories in Bordeline Personality Disorder. Behav Res Ther 40: 1063-8, 2002.

Beck, A.T. - Beyond Belief: A Theory of Modes, Personality, and Psychopathology, In Salkovskis, P.M. (ed.). Frontiers of Cognitive Therapy. Guilford Press, New York, 1-25, 1996.

BeCK, A.T.; Alford, B. - O Poder integrador da terapia cognitiva. Artes Médicas, Porto Alegre, 2000. (Original publicado em 1996).

Beck, A.T.; Freeman, A. - Terapia cognitiva dos transtornos de personalidade. Artes Médicas, Porto Alegre, 1993. (Original publicado em 1990).

BECK, A.T.; Rush, A.J.; SHAW, B.F.; EMERY, G. - Terapia cognitiva da depressão. Artes Médicas, Porto Alegre, 1997. (Original publicado em 1979).

Beck, J.S. - Terapia cognitiva: teoria e prática. Artes Médicas, Porto Alegre, 1997. (Original publicado em 1995).

Bootzin, R.R.; McKniGHT, K. N. - The Role of Biased Information Processing in Depression: Evaluation and Implications for Treatment. Behav Ther: 29, 619-30, 1998.

Brittlebank, A.D.; SCOTt, J.; Williams, J.M.G.; Ferrier, I. N. - Autobiographical Memory in Depression: State or Trait Marker? Br J Psychiatry 162: $118-21,1993$

Burke, M.; Mathews, A. - Autobiographical Memory and Clinical Anxiety. Cogn Emotion 6: 23-35, 1992.

DAlgleish, T.; Spinks, H.; Yiend, J.; KuYKen, W. - Autobiographical Memory Style in Seasonal Affective Disorder and its Relationship to Future Symptom Remission. J Abnorm Psychol 110(2): 335-40, 2001.

D’ZuRilla, T.J. - Problem Solving Therapies, In Dobson, K. S. (ed.). Handbook of Cognitive-Behavioral Therapies. Guilford Press, New York, 85-135, 1988 .

EICH, E.; Schooler, J.W. - Cognition/Emotion Interactions, In Eich, E.; Kihlstrom, J.F.; Bower, G.H.; Forgas, J.P.; Niedenthal, P.M. (eds.) Cognition and Emotion. Oxford University Press, New York, 3-29, 2000.

Evans, J.; Williams, J.M.G.; O’Loughlin, S.; Howells, K. - Autobiographical Memory and Problem Solving Strategies of Parasuicide Patients. Psychol Med 22: 399-405, 1992.

Eysenc, M.W.; KeANe, M.T. - Psicologia cognitiva: um manual introdutório. Artes Médicas, Porto Alegre, 1994. (Original publicado em 1989).

Goddard, L.; Dritschel, B.; BuRTon, A. - Role of Autobiographical Memory in Social Problem Solving and Depression. J Abnorm Psychol 105: 609-16, 1996.

Harvey, A.G.; Bryant, R. A.; Dang, S.T. - Autobiographical Memory in Acute Stress Disorder. J Consult Clin Psychol 66: 500-6, 1998

Healy, H.; Williams, M.G. - Autobiographical Memory. In Dalgleish, T.; Power, M. (eds.). Handbook of Cognition and Emotion. John Wiley; Sons, New York, 229-42, 1999.

Jones, J.L. - The Psychotherapist's Guide to Human Memory. Basic Books, New York, 1999.

Lyubomirsky, S.; Nolen-Hoeksema, S. - Effects of Self-focused Rumination on Negative Thinking and Interpersonal Problem Solving. J Pers Soc Psychol 69: 176-90, 1995.

McNally, R.J.; Lasko, N.B.; Macklin, M.L.; PITMAN, R.K. - Autobiographical Memory Disturbance in Combat-related Posttraumatic Stress Disorder. Behaviour Research and Therapy 33: 629-30, 1995.

McNally, R.J.; Litz, B.T.; Prassas, A.; Shin, L.M.; Weathers, F.W. - Emotional Priming of Autobiographical Memory in Post-traumatic Stress Disorder. Cogn Emotion 8: 351-67, 1994.

Neufeld, C.B.; Stein, L.M. - A compreensão da memória segundo diferentes perspectivas teóricas. Rev Est Psicologia 18: 50-63, 2001.

Nezu, A.M.; Nezu, C.M. - Problem Solving Therapy. J Psychother Integr 11: 187-205, 2001

Nolen-Hoeksema, S. - Responses to Depression and their Effects on the Duration of Depressive Episodes. J Abnorm Psychol 100: 569-82, 1991.

Peeters, F.; Wessel, I.; Merckelbach, H.; Boon-Vermeeren, M. - Autobiographical Memory Specificity and The Course of Major Depressive Disorder. Compr Psychiatry 43(5): 344-50, 2002.
Pollock, L.R.; Williams, J.M.G. - Problem Solving and Suicidal Behavior. Suicide Life Threat Behav 28: 375-87, 1998.

Pollock, L.R.; Williams, J.M.G. - Effective Problem Solving in Suicide Attempters Depends on Specific Autobiographical Recall. Suicide Life Threat Behav 31, 386-96, 2001.

SCHACTER, D.L. - The Seven Sins of Memory: How the Mind Forgets and Remembers. Houghton Mifflin Company, New York, 2001.

Sterneerg, R.J. - Psicologia cognitiva. Artes Médicas, Porto Alegre, 2000. (Original publicado em 1996).

Tulving, E.; Lepage, M. - Where in the Brain is the Awareness of One's Past? In Schacter, D. L.; Scarry, E. (eds.). Memory, Brain and Belief. Harvard University Press, Cambridge, 208-28, 2000.

WAINER, R. - Parâmetros cognitivos e afetivos de um modelo da depressão em adultos femininos. Dissertação de Mestrado não-publicada, Faculdade de Psicologia, Pontifícia Universidade Católica do Rio Grande do Sul, Porto Alegre, 1997.

WAINER, R. - Os processos inferenciais nos diálogos psicoterapêuticos correlações entre a Semântica do Dito e a Pragmática do Comunicado. Tese de Doutorado não-publicada, Faculdade de Psicologia, Pontifícia Universidade Católica do Rio Grande do Sul, Porto Alegre, 2002

Wainer, R.; Pergher, G.K.; Piccoloto, N.M. - Psicologia e terapia cognitiva: contribuições da pesquisa experimental para prática psicoterápica, In Knapp, W.P. (ed.). TCC na prática: fundamentos científicos e técnicos das terapias cognitivo-comportamentais. Artes Médicas, Porto Alegre, no prelo.

Wainer, R.; Pergher, G.K.; Piccoloto, N.M. - Terapia cognitivocomportamental das depressões, In Caminha, R.; Wainer, R.; Oliveira, M.; Piccoloto, N. (eds.). Psicoterapias cognitivocomportamentais: teoria e prática. Casa do Psicólogo, São Paulo, 61-85, 2003.

WATKInS, E.; TEASDALE, J.D. - Rumination and Overgeneral Memory in Depression: Effects of Self-focus and Analytic Thinking. J Abnorm Psychol 110: 353-7, 2001.

Watkins, E.; Teasdale, J.D.; Williams, R.M. - Decentring and Distraction Reduce Overgeneral Autobiographical Memory in Depression. Psychol Med 30: 911-20, 2000.

Wessel, I.; Meeren, M.; Peeters, F.; Arntz, A.; Merckelbach, H. - Correlates of Autobiographical Memory Specificity: The Role of Depression, Anxiety and Childhood Trauma. Behav Res Ther 39: 409-21, 2001.

Wilhelm, S.; McNally, R.J.; Baer, L.; Florin, I. - Autobiographical Memory in Obsessive-Compulsive Disorder. Br J Clin Psychol 36: 21-31, 1997.

Williams, J.M.G. - Autobiographical Memory and Emotional Disorders, In Christianson, S. A. (ed.). The Handbook of Emotion and Memory: Research and Theory. Lawrence Erlbaum Associates, Hillsdale, 45177, 1992.

Williams, J.M.G. - Memory Processes and Psychotherapy. In Salkovskis, P. M. (ed.). Frontiers of Cognitive Therapy. Guilford Press, New York, 97-113, 1996.

Williams, J.M.G.; Broadbent, K. - Autobiographical Memory in Suicide Attempters. J Abnorm Psychol 95: 144-9, 1986.

Williams, J.M.G.; Wells, J. - Pacientes suicidas. In Scott, J.; Williams, J.M.G.; Beck, A.T. Terapia cognitiva na prática clínica: um manual prático. Artes Médicas, Porto Alegre, 253-78, 1994. (Original publicado em 1992).

Williams, J.M.G.; Eluis, N.C.; Tyers, C.; Healy, H.; Rose, G.; Macleod, A.K. The Specificity of Autobiographical Memory and Imageability of the Future. Mem Cognit 24: 116-25, 1996.

Williams, J.M.G.; Teasdale, J.D.; Segal, Z.V.; Soulsby, J. - Mindfulness-Based Cognitive Therapy Reduces Overgeneral Autobiographical Memory in Formerly Depressed Patients. J Abnorm Psychol 109: 150-5, 2000 .

Williams, J.M.G.; Watts, F.N.; Macleod, C.; Mathews, A. - Cognitive Psychology and the Emotional Disorders. (2. ed.). John Wiley; Sons, Chichester, 1997.

Young, J.E.; Beck, A.T.; Weinberger, A. - Depressão. In Barlow, D. H. (ed.) Manual clínico dos transtornos psicológicos. (2. ed.). Artes Médicas Porto Alegre, 274-312, 1999. (Original publicado em 1993). 